\title{
Comparison of the Activity and Proprioception of Trunk Muscles According to Different Types of Bridge Exercises in Subjects with and without Chronic Low Back Pain
}

\author{
Yong-Soo Kong, Yoon-Tae Hwang \\ Department of Physical Therapy, Gangneung Yeongdong College, Gangneung, Korea
}

\begin{abstract}
Purpose: The purpose of the present study was to examine joint position senses and muscle activity in subjects with and without chronic low back pain and to determine the effects of different types of bridge exercises on their trunk muscle activity.

Methods: Thrity -eight subjects with chronic low back pain and thrity healthy controls participated in the experiment. Joint position senses and trunk muscle activity levels were measured during the different bridge exercise methods.

Results: The joint position senses of the healthy group and chronic low back pain group showed significant differences during lumbar flexion, lumbar extension, lumbar lateral flexion, and lumbar rotation. The muscle activity levels of the transversus abdominis (TrA), internal oblique (IO), and external oblique (EO) were highest in the prone bridge exercise (PBE) group, followed by the supine bridge swiss ball exercise (SBSE) group and supine bridge exercise (SBE) group in order of precedence. The muscle activity level of the erector spinae (ES) was highest in the SBSE group, followed by the SBE and PBE groups in order of precedence.

Conclusion: Overall, the results suggest that chronic low back pain is associated with declines in joint position senses and that PBEs increase trunk muscle activity more than conventional bridge exercises.
\end{abstract}

Keywords: Bridge exercise, Muscle activity, Proprioception

\section{INTRODUCTION}

Low back pain is one of the most common and costly diseases in modern society with $60-80 \%$ of people experiencing such pain at least once in their lifetime. ${ }^{1}$ Although most cases are resolved, some people (5-5\%) suffer persistent pain, without any response to treatment. ${ }^{2}$ In addition, patients with low back pain show decreased overall physical activity due to pain, structural damage, and suppression of the reflexive muscular contraction mechanism. Longterm inactivity and disuse induce muscular atrophy and muscle weakness, leading to further exacerbation of low back pain, secondary spinal damage, and physical disabilities. ${ }^{3}$

There are various causes of low back pain. These include structural factors resulting from lesions in the spine and diseases in any organ in the abnormal cavity or reproductive organs. Psychological

Received Nov 14, 2015 Revised Dec 15, 2015

Accepted Dec 24, 2015

Corresponding author Yong-Soo Kong

E-mail twinkong@hanmail.net factors, such as stress and hysteria (females), and mechanical hypofunction of the musculoskeletal system are also implicated in low back pain. Weakening of the muscles and ligaments around the spine due to insufficient exercise is a major cause of low back pain. ${ }^{4}$ In addition, patients with low back pain reduce their physical activities to reduce the pain caused by the performance of daily living activities, ${ }^{5}$ and this causes hypofunction and low self-regard. ${ }^{6}$

As low back pain progresses toward a chronic state, it restricts the range of lumbar and joint motions, secondarily leading to trunk muscle weakening, decreased endurance and flexibility, ${ }^{7,8}$ and reduced physical activity. When the pain become chronic, the crosssectional areas of the muscles around the spine decrease, resulting in disuse atrophy. Therefore, chronic low back pain has adverse effects on home and social lives. It may also be associated with economic difficulties and affect interpersonal relationships. It has been

Copylight (C2015 The Korea Society of Physical Therapy

This is an Open Access article distribute under the terms of the Creative Commons Attribution Non-commercial License (Http:// creativecommons.org/license/by-nc/3.o.) which permits unrestricted non-commercial use, distribution, and reproduction in any medium, provided the original work is properly cited. 
reported that patients with chronic low back pain (CLBP) experience confusion in independent life and self-images and many related sufferings. ${ }^{10}$

In many patients with CLBP, decreased mobility results in reduced lumbar flexibility, muscle strength, and muscle endurance. The degradation of lumbar function may lead to chronic low back pain. Lumbar muscle strengthening is essential for the relief of low back pain in cases of pain and lumbar muscle fatigue. ${ }^{11}$ The symptoms of low back pain include paresthesia, radiating pain in the lower extremities, reduced muscle strength, reduced range of joint motions, and asymmetry between the left and right side. ${ }^{12}$ Low back pain causes problems in the senses of joint positions and motions; senses of acting force, action force, and heaviness; and senses of recognized timing of muscle contraction, ${ }^{13}$ leading to consequent damage to proprioceptive senses. ${ }^{14}$ In addition, proprioceptor disorders associated with low back pain give rise to chronic pain and restrictions in the range of joint motions. ${ }^{13}$

Prescribing appropriate exercises for chronic low back pain patients and diagnosing the pain syndrome are very important in physical therapy programs. ${ }^{15}$ Recent studies of elite athletes highlighted the importance of lumbar stabilization exercises for training deep muscles to prevent damage to the intervertebral disks, small joints, and other surrounding tissues and to minimize the stress imposed on the spinal column while performing at a high level. ${ }^{16,17}$ Lumbar stabilization exercises improve the stability of the spine and pelvis during functional postures and movements, ${ }^{18}$ recover the ability to control muscles and movements and balance, and enhance trunk and deep muscle strength to promote health. They are used both as rehabilitation exercises for low back pain patients and as training exercises for athletes. ${ }^{19}$

Among lumbar stabilization exercises, bridge exercises are frequently used by clinics because they are performed in positions where low back pain patients feel comfortable and feel less pain. Bridge exercises retrain gross muscles and fine muscles so that they can coordinate at appropriate ratios and can stabilize trunk muscles and strengthen hip and lower extremity muscles. ${ }^{20}$ Researchers reported that deep abdominal muscle training methods could be applied, in addition to bridge exercises, to strengthen weakened trunk muscles by increasing the muscle activity of deep muscles. Bjerkefors et al. ${ }^{21}$ and $\mathrm{Kim}^{22}$ reported that the use of balls during bridge exercises greatly improved trunk muscle activity.
The aim of the present study was to analyze the trunk joint position senses of subjects with and without chronic low back pain and to determine the effects of the trunk joint position senses on trunk muscle activity to propose effective bridge exercise methods.

\section{METHODS}

\section{Study subjects}

The study group consisted of 30 healthy subjects (16 males, 14 females) and 38 patients with chronic low back pain (13 males, 25 females) who were receiving physical therapy at $\mathrm{P}$ hospital. The mean age, weight, and height of those in the healthy group was 38.4 years, $64.1 \mathrm{~kg}$, and $167.3 \mathrm{~cm}$, respectively. The mean age, weight, an height of those in the CLBP patient group was 41.4 years, $61.8 \mathrm{~kg}$, and 164.2 $\mathrm{cm}$, respectively.

Before starting the experiment, all participants were required to read and sign an informed consent form, in accordance with the ethical standards of the Declaration of Helsinki. The protocol for this study was approved by the local ethics committee. The experimental group was randomly selected. The selection criteria were as follows: The healthy subjects were selected from those who had no medical history of functional disorders of the nervous system or musculoskeletal system, vertigo, or other balance disorders. The chronic low back pain patients were selected from patients in their 20-50s who had CLBP for at least 6 months and who had been diagnosed with CLBP using various examinations, such as X-rays, CT, and/or MRI. Those who had problems in their vestibular organs or neural damage, those who were taking balance-related drugs, those who had undergone spinal surgery due to orthopedic problems, and those who could not perform the exercise program were excluded from the experiment.

\section{Experimental method}

The general characteristics, medical history, and disease names were written firsthand by the study subjects using questionnaires, and the CLBP group was randomly divided into three exercise groups:

Supine bridge exercise (SBE): Lying down, the arms are spread at approximately $30^{\circ}$ and the knees are flexed at $90^{\circ}$; the palms are placed face down on the ground, and the head and neck are kept straight with the eyes looking at the ceiling. Supine bridge on Swiss ball exercise (SBSE): With an identical starting position to Exercise 1, 
the feet are placed on a Swiss ball. Prone bridge exercise (PBE): In the prone position, the body weight is supported on the forearms and the toes, with the elbows flexed at $90^{\circ}$, the neck slightly extended, and the eyes looking forward.

\section{Procedure}

A Zebris (Zebris Medical GmbH CMS100, Germany) was used to examine proprioceptive senses. The Zebris is a device for measurement of the spinal range of motion, and it has been shown to have high stability and reliability. Two sensors were used to measure the spinal joint angles detected by the sensors using ultrasonic waves. To measure the proprioceptive senses, joint position senses were measured. First, the patients were instructed to maintain a neutral position. Then, flexion-extension, right rotation, and right lateral flexion were measured in a standing position. The patients were instructed to move while maintaining a neutral position, keeping their eyes open to enable them to recognize angles. Thereafter, they were instructed to find arbitrary angles with their eyes closed. The patients were instructed to shout "stop" when they found the arbitrary angles. During the measurement, the patients received no visual feedback from the monitor, and each motion was limited to three times to control learning effects. In each case, the difference between an actual angle and an arbitrary angle was measured three times, and the average value was calculated.

A wireless electromyography (EMG) system (Telemyo 2400T-G2, Noraxon, USA) was used to examine trunk muscle activity. The EMG system was connected to a bipolar snap electrode consisting of a ground electrode, an active electrode, and a reference electrode, which was used as a measuring electrode. The surface digital-processed surface EMG signals were processed using a personal computer using the MR-XP program. The sampling rate of the surface EMG signals was $1,024 \mathrm{~Hz}$, and 20-500 Hz band pass filters and 60 $\mathrm{Hz}$ notch filters were used in the sampling. The collected signals were full-wave rectified and processed into root mean squares for analysis. The electrode attachment sites were rubbed with fine sandpaper 3-4 times to reduce skin resistance by removing dead skin cells. Before attaching the electrodes, the surface of the skin was cleaned using cotton wool wetted with rubbing alcohol. The electrodes were attached along the muscle fiber running direction, and the ground electrode was attached above the anterior superior iliac spine.

The trunk muscle activity levels according to the bridge exercise methods were measured by attaching the electrodes to four muscles: transverse abdominis (in the middle between the navel line and anterior superior iliac spine, and at the central lateral point of the pubic bone), internal oblique (in the middle between the navel line and anterior superior iliac spine), external oblique (a point $15 \mathrm{~cm}$ lateral to the navel), and erector spinae (a point $2 \mathrm{~cm}$ lateral to the $\mathrm{L} 2 \mathrm{spi}$ nous process) on the dominant side of the experimental subjects. ${ }^{23}$

The maximal voluntary isometric contraction was used to standardize the action potential of each muscle. The method described by Kendall et al. ${ }^{24}$ was used to determine the maximal voluntary isometric contraction. In each motion, the posture was held for 10 sec. To reduce errors at the beginning and end, the measured values for $5 \mathrm{sec}$, excluding the first $3 \mathrm{sec}$ and the last $2 \mathrm{sec}$, were used to analyze the muscle activity. The EMG values were measured three times during all the motions, and the average values were calculated.

\section{Data analysis}

The collected data were statistically processed using SPSS 18 PASW Statistics, and descriptive statistics were used to determine the general characteristics of the subjects. Shapiro-Wilk tests were conducted to test intergroup normality, and independent t-tests were conducted to determine the joint position senses and muscle activity of the healthy group and CLBP group. In addition, one-way ANOVA were conducted to detect differences among the CLBP

Table 1. Characteristics of between CLBP groups $(\mathrm{N}=38)$

\begin{tabular}{|c|c|c|c|c|c|}
\hline Characteristics & Total & SBE & SBSE & PBE & $p$ \\
\hline Age (yr) & $41.47 \pm 8.38$ & $39.45 \pm 7.75$ & $41.92 \pm 10.07$ & $42.69 \pm 7.15$ & 0.633 \\
\hline Height (cm) & $164.11 \pm 6.21$ & $163.54 \pm 5.29$ & $163.71 \pm 6.86$ & $165.18 \pm 6.56$ & 0.784 \\
\hline Weight (kg) & $61.78 \pm 8.47$ & $60.9 \pm 7.55$ & $59.5 \pm 6.95$ & $63.9 \pm 10.18$ & 0.227 \\
\hline Sex Male & $13(34.2)$ & $4(36.4)$ & $4(28.6)$ & $5(38.5)$ & 0.861 \\
\hline Female & $25(65.8)$ & $7(63.6)$ & $10(71.4)$ & $8(61.5)$ & \\
\hline
\end{tabular}

aMean \pm standard deviation.

SBE: Supine bridge exercise, SBSE: Supine bridge on swiss ball exercise, PBE: Prone bridge exercise. 
group according to the bridge exercise methods. The statistical significance level was set to $\mathrm{p}<0.05$.

\section{RESULTS}

The general characteristics of the CLBP group according to the bridge exercise methods are presented in Table 1. The joint position senses of the healthy group and CLBP group during lumbar flexion, extension, lateral flexion, and rotation showed statistically signifi-

Table 2. Comparison of joint position sense error between CG and CLBPG

(Unit: degree)

\begin{tabular}{lccc}
\hline Parameter & CG & CLBPG & $t$ \\
\hline Flexion & $2.49 \pm 0.78$ & $4.96 \pm 1.66$ & $8.075^{*}$ \\
Extension & $1.99 \pm 0.87$ & $2.71 \pm 0.81$ & $3.508^{*}$ \\
Side-bending & $1.85 \pm 0.83$ & $3.33 \pm 1.58$ & $4.915^{*}$ \\
Rotation & $1.37 \pm 0.59$ & $2.34 \pm 0.87$ & $5.375^{*}$ \\
\hline
\end{tabular}

CG: Control group, CLBPG: Chronic low back pain group. ${ }^{*} \mathrm{p}<0.001$.

Table 3. Comparison of muscle activities of betweens groups

(Unit: \%MVIC)

\begin{tabular}{cccll}
\hline & Exercise & \multicolumn{1}{c}{ CG } & \multicolumn{1}{c}{ CLBPG } & \multicolumn{1}{c}{$\mathrm{t}$} \\
\hline IO & SBE & $5.32 \pm 2.39$ & $11.98 \pm 4.13$ & $4.99^{* *}$ \\
& SBSE & $12.16 \pm 5.96$ & $15.33 \pm 7.42$ & $2.99^{*}$ \\
& PBE & $35.87 \pm 9.10$ & $58.47 \pm 20.98$ & $3.73^{*}$ \\
TrA & SBE & $9.38 \pm 5.09$ & $13.53 \pm 7.30$ & $2.05^{*}$ \\
& SBSE & $6.20 \pm 2.96$ & $18.80 \pm 8.48$ & $4.43^{* *}$ \\
& PBE & $30.92 \pm 9.73$ & $48.10 \pm 18.14$ & $3.21^{*}$ \\
EO & SBE & $5.74 \pm 2.83$ & $11.62 \pm 4.76$ & $4.84^{* *}$ \\
& SBSE & $7.02 \pm 2.49$ & $15.57 \pm 5.80$ & $5.28^{* *}$ \\
& PBE & $27.61 \pm 10.60$ & $46.24 \pm 13.89$ & $4.81^{* *}$ \\
ES & SBE & $32.29 \pm 9.18$ & $45.90 \pm 14.58$ & $2.89^{*}$ \\
& SBSE & $35.18 \pm 12.26$ & $48.81 \pm 15.96$ & $3.11^{*}$ \\
& PBE & $6.90 \pm 2.28$ & $10.43 \pm 4.40$ & $2.08^{*}$ \\
\hline
\end{tabular}

CG: Control group, CLBPG: Chronic low back pain group, SBE: Supine bridge exercise, SBSE: Supine bridge on swiss ball exercise, PBE: Prone bridge exercise, IO: Internal oblique, TrA: Transverse abdominis, EO: External oblique, ES: Erector spinae.

${ }^{*} p<0.05,{ }^{* *} p<0.001$. cant differences $(\mathrm{p}<0.05)$ (Table 2). The trunk activity of the healthy group was significantly different compared to that of the CLBP group $(\mathrm{p}<0.05)$ (Table 3). Among the CLBP group according to the bridge exercise methods, the highest levels of muscle activity of the $\mathrm{IO}, \mathrm{EO}$, and TrA were recorded in the PBE group, followed by the SBSE and SBE groups in order of precedence. The muscle activity of the ES was highest in the SBSE group, followed by the SBE group and PBE group in order of precedence. According to the results of post-hoc tests, the muscle activity levels of the IO, EO, TrA, and EO were significantly difference in between the SBE and PBE groups and between the SBSE and PBE groups $(\mathrm{p}<0.05)$ (Table 4).

\section{DISCUSSION}

This study was consisted of two experiments. First, proprioception and muscle activation level were compared between chronic low back pain group and control group. Then, muscle activation level of chronic low back pain group was measured during performing different variations of the bridge exercise for strengthening trunk muscles. For the proprioception comparison, joint position sensation was measured. According to the results of the study, joint position sense during lumbar flexion were $2.49^{\circ}$ in the healthy group and $4.96^{\circ}$ in the CLBP group. Joint position sense during lumbar extension were $1.99^{\circ}$ in the healthy group and $2.71^{\circ}$ in the CLBP group. Joint position sense during lumbar lateral flexion were $1.85^{\circ}$ in the healthy group and $3.33^{\circ}$ in the CLBP group. In the healthy group, joint position sense during lumbar rotation were $1.37^{\circ}$, whereas they were $2.34^{\circ}$ in the CLBP group. Thus, there were significant differences in joint position sense during all motions between the healthy group and CLBP group. Therefore, there were differences in the proprioceptive senses of the healthy group and CLBP group. Furthermore, the joint position sense and postural control abilities of those with CLBP were inferior compared to those of the healthy

Table 4. Comparison of the activities in different types of bridge exercises

(Unit: \%MVIC)

\begin{tabular}{lccccc}
\hline & SBE (A) & SBSE (B) & PBE (C) & F & post-hoc \\
\hline IO & $11.98 \pm 4.13$ & $15.33 \pm 7.42$ & $58.47 \pm 20.98$ & $48.47^{* *}$ & AB/C \\
TrA & $13.53 \pm 7.30$ & $18.80 \pm 8.48$ & $48.10 \pm 18.14$ & $28.16^{* *}$ & AB/C \\
EO & $11.62 \pm 4.76$ & $15.57 \pm 5.80$ & $46.24 \pm 13.89$ & $53.28^{* *}$ & AB/C \\
ES & $45.90 \pm 14.58$ & $48.81 \pm 15.95$ & $10.43 \pm 4.40$ & $36.51^{* *}$ & $\mathrm{AB} / \mathrm{C}$ \\
\hline
\end{tabular}

SBE: Supine bridge exercise, SBSE: Supine bridge on swiss ball exercise, PBE: Prone bridge exercise, IO: Internal oblique, TrA: Transverse abdominis, EO: External oblique, ES: Erector spinae. ${ }^{*} p<0.05,{ }^{* *} p<0.001$. 
subjects.

Declines in proprioceptive senses reduce neuromuscular control ability and cause functional instability of the body, and this physical instability leads to repetitive damage, resulting in a continuous vicious circle. ${ }^{25}$ In the present study, the proprioceptive senses of the CLBP group were much lower than those of the healthy group. In particular, large between-group differences were apparent during lumbar flexion. These results are consistent with those of Field et al. ${ }^{26}$ who found that the ability of a low back pain group to return to a standing position taken seconds earlier was poorer than that of a healthy group without low back pain. The same study found that the low back pain group showed a greater tendency to pass or fall short of the location of the previous position when loads were applied to their bodies.

Methods for controlling low back pain include drug therapy, physical therapy, and exercise therapy. Drug therapy provides temporary pain relief but is costly. Exercises are a cost-effective and effective method for chronic pain relief and recurrence prevention. ${ }^{27}$ Recent studies and treatments aimed at preventing low back pain and reducing the possibility of recurrence have focused on improving the stability of the trunk. This involves correcting the alignment and posture of the vertebral column, and small unit muscles involved in trunk stability that are mainly located deep in the body. ${ }^{28}$

Trunk stabilization exercises in cases of insufficient muscle strength, flexibility, balance, and agility are aimed at muscle strengthening and the recovery of the ability to control muscles, movements, balance, and agility. ${ }^{29}$ Diverse exercise programs have been developed for lumbar stabilization. Among these, low back pain patients were reported to feel comfortable and to feel reduced pain when performing bridge exercises, which retrained gross muscles and fine muscles so that they can coordinate at appropriate ratios. ${ }^{30}$ It was reported that low back pain patients had lower muscle strength in their transversus abdominis, erector spinae, and other abdominal muscles compared to healthy subjects. ${ }^{31}$ Also, it was reported that the weakness of trunk muscles, the internal and external oblique, transverse abdominis, and multifidus was main reason for chronic low back pain. ${ }^{32}$

In this study, the differences of trunk muscles activation levels between chronic low back pain group and control group during different bridge exercises for muscle strengthening program were measured. Compared to the healthy group, the CLBP group, which consisted of the SBE, SBSE, and PBE groups, showed significantly lower levels of activity of the IO, TrA, EO, and ES. The levels of muscle activity of the IO, TrA, and EO increased the most in the PBE group, followed by the SBSE and SBE groups in order of precedence. In both the healthy group and CLBP group, the levels of muscle activity of the ES increased the most in the SBSE group, followed by the SBE group and PBE group in order of precedence In both the healthy group and CLBP group. Also, the activities of IO, TrA, and EO of patients with CLBP showed that more increase was in the PBE group.

Given these results, it can be said that PBEs induce higher levels of muscle activity than conventional bridge exercises or bridge exercises that involve the use of balls because PBEs require the mobilization of more muscles to maintain trunk stability. Kong et al. ${ }^{33}$ reported that PBEs increased muscle activity due to somatologic demands in the process of overcoming instability resulting from the decreased base of support by bearing the body weight with the elbow and toe in distal regions. The results of the present study, which indicated that PBEs performed by healthy subjects to improve trunk stability were more useful than conventional bridge exercises in reducing the muscle activity of the erector spinae while increasing the muscle activity of the rectus abdominis, internal oblique, and external oblique, are consistent with those of an earlier study.

Bridge exercises are useful for developing pelvic muscles and strengthening the lower part of the spine and hip joint extensor muscle to prepare for the stance phase in gaits. They can also selectively increase lower extremity muscle activity while maintaining trunk stability. ${ }^{34}$ In addition, in bridge exercises, the body weight is supported by the elbows. As the loads imposed on the upper arm are reduced, the exercise postures can be maintained for a long time. Thus, they are easier than push-up exercises, and they can be performed by patients with low back pain, even at the beginning of rehabilitation. $^{35}$

Both increasing activation levels of deep muscles like the multifidus and transverse abdominis and decreasing activation levels of superficial muscles like rectus abdominis and erector spinae are needed to maximize spinal stability. ${ }^{36}$ Strengthening the transverse abdominis and the internal oblique is essential, too. ${ }^{30}$ Supine bridge exercise was proved for increasing the erector spinae activation level, not the activation levels of the transverse abdominis, internal and external oblique which are deep muscles. However, prone bridge ex- 
ercises significantly increased not only the transverse abdominis but also the internal and external oblique. Based on these findings, the prone bridge exercise can be used to control posture and alleviate low back pain in chronic low back pain patients with strengthening trunk muscles.

Therefore, it is more beneficial for low back pain patients if the prone bridge exercises and the supine bridge exercises at the same time to improve spinal stability with maximizing posture control and muscle strength as well as for preventing low back pain. Studies that require intervention periods to see changes in proprioceptive senses and muscle activity are supplemented, such studies could be very helpful to chronic low back pain patients.

\section{REFERENCES}

1. O'Sullivan PB. Lumbar segmental 'instability': clinical presentation and specific stabilizing exercise management. Man Ther. 2000;5:2-12.

2. Craig L. Rehabilitation of the spine. 2000: Lippincott; Williams \& Wilkins.

3. Kader DF, Wardlaw D, Smith FW. Correlation between the MRI changes in the lumbar multifidus muscles and leg pain. Clin Radiol. 2000;55: 145-9.

4. Cailliet R. Low back pain syndrome(4thed). F.A Davis Company, 1992, Philadelphia: 116-46.

5. Picavet HS, Schuit AJ. Physical inactivity: a risk factor for low back pain in the general population? J Epidemiol Community Health. 2003;57: 517-8.

6. Crombez G, Vlaeyen JW, Heuts PH, et al. Pain-related fear is more disabling than pain itself: evidence on the role of pain-related fear in chronic back pain disability. Pain. 1999;80:329-39.

7. Faas A: Exercises: which ones are worth trying, for which patients, and when? Spine. 1996;21:2878-9.

8. Koumantakis GA, Watson PJ, Oldham JA. Trunk muscle stabilization training plus general exercise versus general exercise only: randomized controlled trial of patients with recurrent low back pain. Physical Therapy. 2005;85:209-25.

9. Yoshihara K, Shirai Y, Nakayama Y, et al. Histochemical changes in the multifidus muscle in patients with lumbar intervertebral disk herniation. Spine. 2001;26:622-6.

10. Kerns RD, Rosenberg R, Jacob MC. Anger expression and chronic pain. J Behav Med. 1994;17:57-67.

11. Kankaanpaa M, Colier WN, Taimela S, et al. Back extensor muscle oxygenation and fatigability in healthy subjects and low back pain patients during dynamic back extension exertion. Pathophysiology. 2005;12: 267-73.

12. Kim SY. Comparison of the difference of weight-bearing distribution between subjects with low back pain and healthy subjects. Phys ther kor. 2001; 8:1-8.

13. Comerford MJ, Mottram SL. Functional stability re-training: principles and strategies for managing mechanical dysfunction. Man Ther. 2001;6: 3-14.

14. Kofotolis N, Kellis E. Effects of two 4-week proprioceptive neuromuscular facilitation programs on muscle endurance, flexibility, and functional performance in women with chronic low back pain. Phys Ther. 2006;86: 1001-12.

15. Shweta A, Sarvaiya-shah BS. Interrater reliability of the movemnet impairment clssification for lumbar spine syndromes in patients with chronic low back pain., 2005: University of Texas woman's. USA.

16. Luoto S, Taimela S, Alaranta H et al. Psychomotor speed in chronic lowback pain patients and healthy controls: construct validity and clinical significance of the measure. Percept Mot Skills. 1998;87:1283-96.

17. Elia DS, Bohannon RW, Cameron D et al. Dynamic pelvic stabilization during hip flexion: a comparison study. J Orthop Sports Phys Ther. 1996;24:30-6.

18. Richardson CA, Snijders CJ, Hides JA et al. The relation between the transversus abdominis muscles, sacroiliac joint mechanics, and low back pain. Spine 2002;27:399-405.

19. Behm DG, Drinkwater EJ, Willardson JM et al. The use of instability to train the core musculature. Appl Physiol Nutr Me. 2010;35:91-108.

20. Kisner C, Colby LA. Therapeutic Exercise: Foundations and Techniques. 2002: 4th ed. Philadelphia. FA Davis.

21. Bjerkefors A, Ekblom M, Josefsson K et al. Deep and superficial abdominal muscle activation during trunk stabilization exercises with and without instruction to hollow. Man Ther. 2010;15:502-7.

22. Kim MJ. Effect of bridging stabilization exercises on trunk muscles activity on and off a swiss ball. Phys Ther Kor. 2009;16:18-24.

23. Carm JR, Kasman GS, Holtz J. Introduction to Surface Electromyography. Gaithersburg, MD. Aspen. 1998:360-74.

24. Kendall FP, McCreary EK, Provance PG et al. Muscles; testing and function with posture and pain. 5th. Lippincott Williams \& Wilkins, 2005.

25. Prentice WE. Rehabilitation techniques in sports medicine. McGrw-Hil, 1999.

26. Field E, Abdel-Moty E, Loudon J. The effect of back injury and load on ability to replicate a novel posture. Journal of Back and Musculoskeletal Rehabilitation. 1997;8:199-207.

27. Mather CG. Effective physical treatement for chronic low back pain. Orthopaedics Clininical North America, 2004;35:57-64.

28. Norris CM. Functional load abdominal training: part 1. J Bodyw Mov Ther. 1999;3:150-8.

29. Standaert CJ, Weinstein SM, Rumpeltes J. Evidence-informed management of chronic low back pain with lumbar stabilization exercises. Spine. 2008;8:114-20.

30. Stevens VK, Coorevits PL, Bouche KG et al. The influence of specific training on trunk muscle recruitment patterns in healthy subjects during stabilization exercises. Man Ther. 2007;12:271-9.

31. Hodges PW. Is there a role for transversus abdominis in lumbo-pelvic stability? Man Ther. 1999;4:74-86.

32. Norris CM. Norris CM. Functional load abdominal training: part 1. Physical Therapy in Sport. 2001:29-39.

33. Kong YS, Cho YH, Park JW. Changes in the activities of the trunk muscles in different kinds of bridging exercises. J Phys Ther Sci. 2013;25: 1609-12.

34. O'Sullivan S, B, Schmitz TJ. Physical rehabilitation: Assessment and 
treatment. 4th ed. 2001:529-64.

35. Ludewig PM, Hoff MS, Osowski EE et al. Relative balance of serratus anterior and upper trapezius muscle activity during push-up exercises.
Am J Sports Med. 2004;32:484-93.

36. Panjabi MM. The stabilizing system of the spine. Part II. Neutral zone and instability hypothesis. J Spinal Disord. 1992;5:390-6; discussion 397. 